\title{
An Electron Microscope Pixel Array Detector as a Universal STEM Detector
}

\author{
David A. Muller ${ }^{1,2}$, Kayla X. Nguyen ${ }^{3}$, Mark W. Tate ${ }^{4}$, Prafull Purohit ${ }^{4}$, Celesta Chang ${ }^{4}$, Michael Cao ${ }^{1}$, \\ Sol M. Gruner ${ }^{4}$, \\ 1. School of Applied and Engineering Physics, Cornell University, Ithaca, NY, USA \\ 2. Kavli Institute for Nanoscale Science, Cornell University \\ 3. Department of Chemistry and Chemical Biology, Cornell University, Ithaca, NY, USA \\ 4. Department of Physics, Cornell University, Ithaca, NY, USA
}

Complete information about the scattering potential of a sample is in principle encoded in the distribution of scattered electrons from a localized beam propagating through it. A new generation of high speed imaging detectors brings us closer to this goal and will allow us to explore practical limits and identify the most promising methods of analysis. We have recently developed an electron microscope pixel array detector (EMPAD) that functions as a compact and high-speed, high dynamic range electron diffraction camera (Figure 1a). It has single electron sensitivity with a signal/noise ratio of 140 for a single electron at $200 \mathrm{keV} \mathrm{[1,2].} \mathrm{It} \mathrm{has} \mathrm{a} \mathrm{dynamic} \mathrm{range} \mathrm{of} 10^{6}$ for primary electrons- i.e a pixel can detect from 1 to 1,000,000 electrons, and reads out an image frame in $0.86 \mathrm{~ms}$. These properties allow us to record essentially an image of all the transmitted electrons, from the unscattered beam to out beyond the HOLZ lines, and do so for every probe position in a real-space, atomic resolution image. Not only does this allow quantitative and simultaneous annular dark and bright field signals on an absolute scale, but from the analysis of the spatially-resolved diffraction patterns we can extract thickness, strain and tilt, octahedral rotations, polarity and even electric and magnetic fields.

As a practical demonstration of dynamic range Figure $1 \mathrm{~b}$ and $1 \mathrm{c}$ show the diffraction patterns generated from $1 \mathrm{~ms}$ and $100 \mathrm{~ms}$ acquisition with a sub-2A beam containing $10 \mathrm{pA}$ probe current at $200 \mathrm{keV}$ in a Tecnai F20. The high-angle scattering is detected with single electron sensitivity simultaneously with the unsaturated high-intensity central beam and crystallographic Bragg discs. Quantitative atomic resolution images from this data are given in [1,2]. Less conventional imaging modes are explored here. Generalized multipole imaging, from the $n^{\text {th }}$ moment of the diffraction pattern directly gives the expectation value of the $\mathrm{n}^{\text {th }}$ moment of the scattered probe's momentum, 牧 $k$ \#). The first moment, or center of mass $(\mathrm{CoM})$ is the probability current flow, which in a thin sample is the beam deflection from the Lorentz force $[3,4]$. Thus, changes in the scattered beam's momentum are directly measured over all angles and a specimen's in-plane magnetic and electric fields can be extracted. In thick samples, the influence of crystal tilt and absolute local sample thickness variations can also be accounted for by recording the full pattern. Fig 2 a shows a $\mathrm{k}^{2}$-weighted diffraction pattern which enhances the Kikuchi bands, isolating crystal tilt. Fig $2 \mathrm{~b}$ shows the measured tilt of domains in multiferroic $\mathrm{BiFeO}_{3}$. From a naïve CoM analysis, this could be incorrectly interpreted as an electric field. Fig $2 c$ shows the true $\mathrm{x}-$ component of the magnetic field in Co imaged by CoM Lorentz-STEM with the familiar magnetic ripple texture of the material.

Fast MMPAD readout allows 4D-STEM imaging at atomic resolution. Example data from our Cscorrected Titan at $120 \mathrm{kV}$ is shown in Fig 3 for $\mathrm{SrTiO}_{3}$. Fig 3 a shows the annular dark field image from

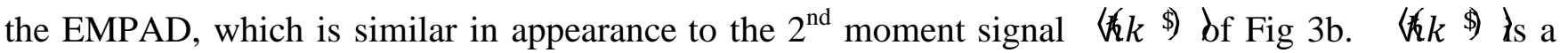
measure of the scattering power of the sample. The CoM images (Fig 3c) give the x\&y derivatives of the potential, which can be obtained by integration [5,6] (Fig 3d), showing the oxygen sublattice clearly, well beyond what the ABF signal provides. [7] 
References:

[1] K. X. Nguyen, et al. Microsc. Microanal. 21 (S3) (2015), 2309

[2] M. W. Tate, et al. Microsc. Microanal. 22, 237-249 (2016).

[3] K. Müller, et al., Nature Communications 5, 1-8 (2014).

[4] A. Lubk and J. Zweck. Physical Review A 91 (2015), 023805

[5] R. Close, Z. Chen, N. Shibata, and S. D. Findlay. Ultramicroscopy 159 (2015), 124-137

[6] I. Lazić, E. G. T. Bosch, and S. Lazar. Ultramicroscopy 160 (2016), 265-280

[7] EM development supported by the NSF MRSEC program (DMR 1120296). Detector development supported by DOE DE-FG02-10ER46693 and the Kavli Institute at Cornell.
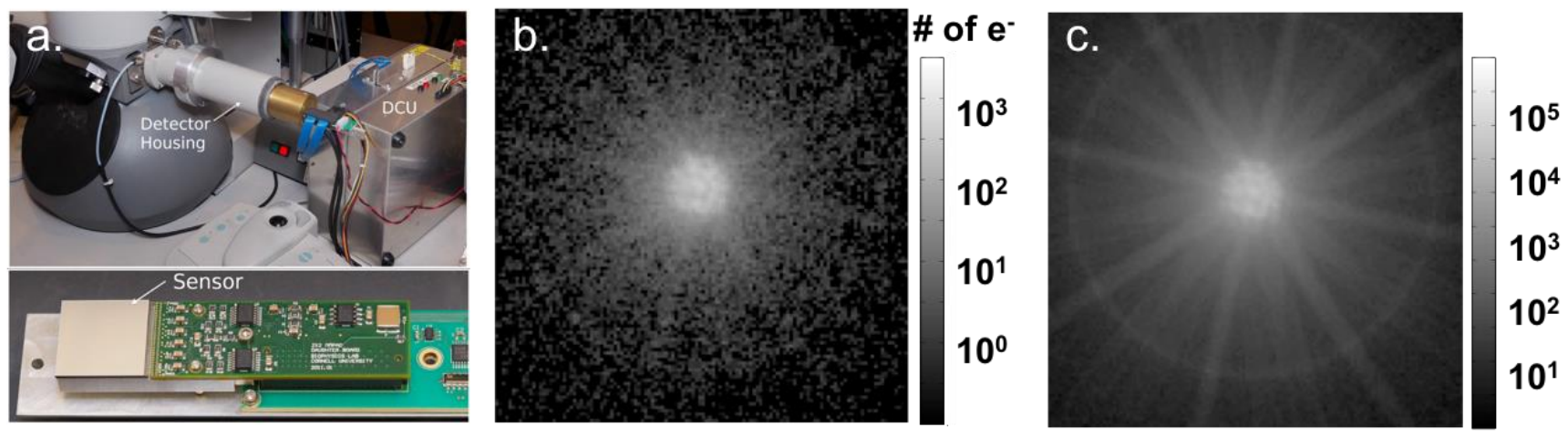

Fig. 1. a) EMPAD detector on FEI F20 showing compact design and sensor. CBED pattern of $\mathrm{BiFeO}$ recorded in b) $1 \mathrm{~ms}$ and c) $100 \mathrm{~ms}$ with $10 \mathrm{pA}$ of beam current. $20 \mathrm{mrad}$ scale bar.[2]
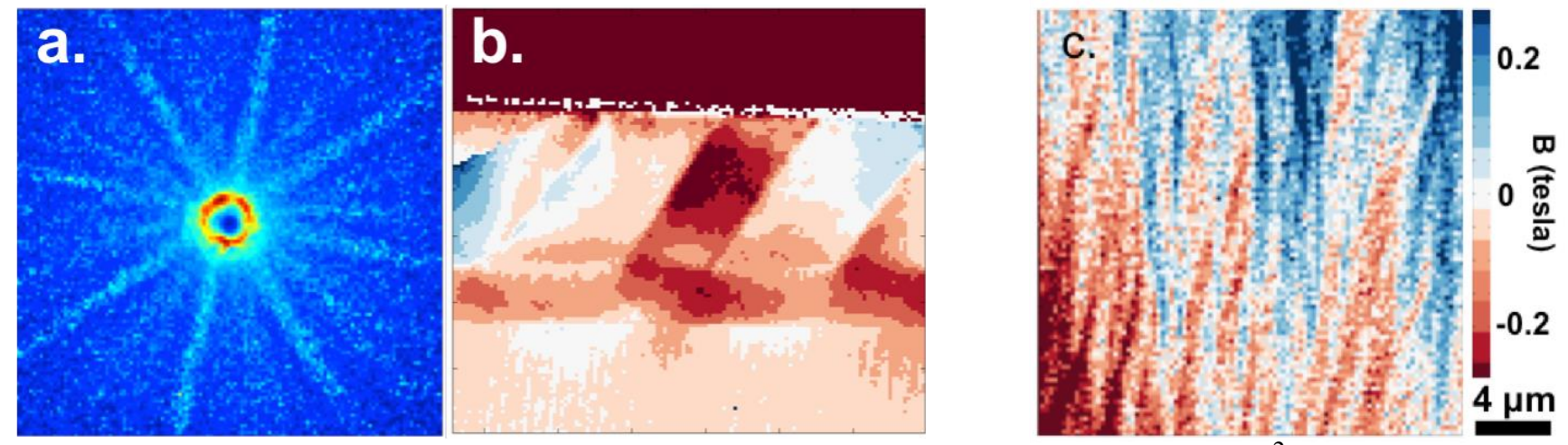

Fig. 2. Mapping crystal tilt from Kikiuchi band shifts in multiferroic $\mathrm{BiFeO}_{3}$. a) $\mathrm{k}^{2}$-weighted diffraction pattern. b) x components of the crystal tilt. (65 sec acquisition). c) Magnetic field ripples in Co thin film mapped in field-free LM-STEM from CoM shifts of the central beam [2]. $65 \mathrm{sec}$ acq.
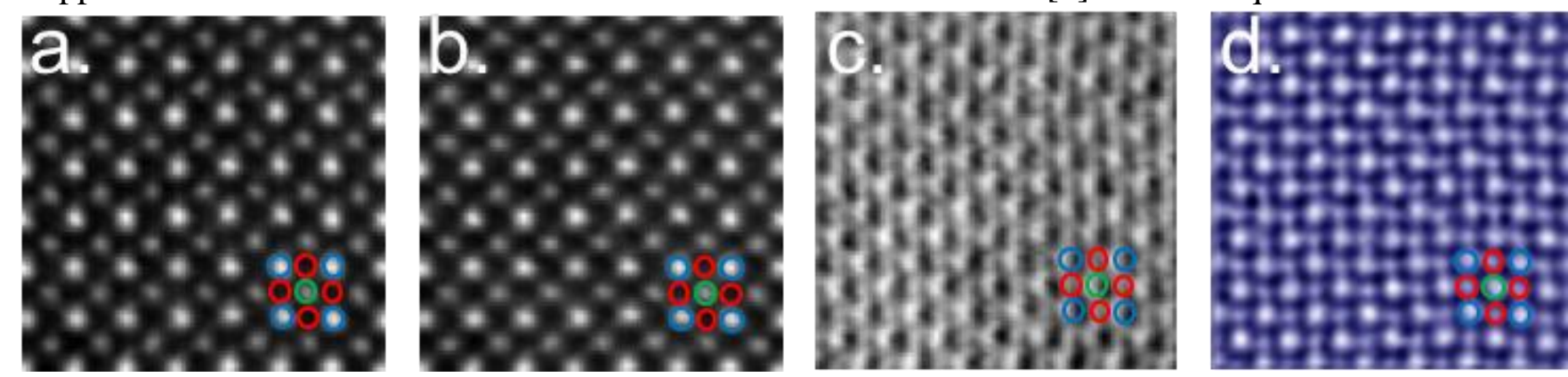

Fig. 3. $120 \mathrm{keV}$ EMPAD STEM data from $\mathrm{SrTiO}_{3}$ : Simultaneously extracted a) ADF, b) Second

Moment, c) First moment 2 ? and d) iCOM image from symmetrized integration of the first moment (c) to derive an effective projected potential, showing the oxygen sublattice. Blue circles- Sr, Red-O, Green- Ti+O. 9 second acquisition time on Cs-corrected Titan Themis 60-300. 